\title{
Patterns of mixed Plasmodium species infections among children six years and under in selected malaria hyper-endemic communities of Zambia: population-based survey observations
}

Lungowe Sitali ${ }^{1,27^{*}}$, James Chipeta ${ }^{2,3}$, John M Miller ${ }^{4}$, Hawela B Moonga ${ }^{5}$, Nirbhay Kumar ${ }^{6}$, William J Moss ${ }^{7}$ and Charles Michelo 2,8

\begin{abstract}
Background: Although malaria is preventable and treatable, it still claims 660,000 lives every year globally with children under five years of age having the highest burden. In Zambia, malaria rapid diagnostic tests (RDTs) that only detect Plasmodium falciparum are the main confirmatory means for malaria diagnosis in most health facilities without microscopy services. As a consequence of this $P$. falciparum species diagnostic approach, non-falciparum malaria is not only under-diagnosed but entirely missed, thereby making the exact disease burden unknown. We thus investigated the prevalence of various Plasmodium spp. and associated burden of infection in selected communities in Zambia.

Methods: Data from two malaria hyper-endemic provinces (Eastern and Luapula) of the 2012 National Malaria Indicator Survey (MIS), conducted between April and May 2012, were used. The MIS is a nationally representative, two-stage cluster survey conducted to coincide with the end of the malaria transmission season. Social, behavioural and background information were collected from households as part of the survey. Thick blood smears, RDTs and dried blood spots (DBS) were collected from children below six years of age. Slides were stained using Giemsa and examined by microscopy while polymerase chain reaction (PCR) was used to analyse the DBS for malaria Plasmodium spp. Multivariate logistic regression was employed to examine the association between background factors and malaria.

Results: Overall, 873 children younger than six years of age were surveyed. The overall prevalence of Plasmodium spp. by PCR was 54.3\% (95\% Cl 51-57.6\%). Of the total Plasmodium isolates, $88 \%$ were $P$. falciparum, $10.6 \%$ were mixed infections and $1.4 \%$ were non-falciparum mono infections. Among the mixed infections, the majority were a combination of $P$. falciparum and P. malariae (6.5\% of all mixed infections). Children two years and older ( $2-5$ years) had three-fold higher risk of mixed malaria infections (aOR $2.8 \mathrm{Cl} 1.31-5.69$ ) than children younger than two years of age.

Conclusion: The high prevalence of mixed Plasmodium spp. infections in this population stresses review of the current malaria RDT diagnostic approaches. The observed less incidence of mixed infections in children under two years of age compared to their older two-to-five-year-old counterparts is probably due to the protective maternal passive immunity, among other factors, in that age group.
\end{abstract}

Keywords: Malaria, Mixed infections, Non-falciparum infections, Prevalence, Zambia

\footnotetext{
*Correspondence: lungowesitali@gmail.com

'Department of Biomedical Science, University of Zambia, School of

Medicine, Lusaka, Zambia

2Department of Paediatrics and Child Health, School of Medicine, Malaria

Research Unit (SMUTH-MRU), Lusaka, Zambia

Full list of author information is available at the end of the article
}

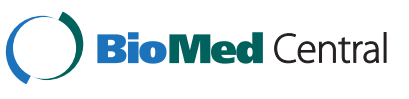

(c) 2015 Sitali et al.; licensee BioMed Central. This is an Open Access article distributed under the terms of the Creative Commons Attribution License (http://creativecommons.org/licenses/by/4.0), which permits unrestricted use, distribution, and reproduction in any medium, provided the original work is properly credited. The Creative Commons Public Domain Dedication waiver (http://creativecommons.org/publicdomain/zero/1.0/) applies to the data made available in this article, unless otherwise stated. 


\section{Background}

Malaria remains a significant public health problem in many countries throughout the world, especially in subSaharan Africa, despite being preventable and treatable [1]. In 2010, the World Health Organisation (WHO) estimated approximately 219 million malaria cases and 660,000 deaths, with $90 \%$ of the deaths occurring in Africa [2]. Approximately $40 \%$ of the world's population lives in areas that have some risk of malaria [3]. The distribution of Plasmodium spp. is not clearly known, although estimates of the global distribution of Plasmodium falciparum and $P$. vivax, the most predominant malaria Plasmodium spp., are widely cited [4-6]. Mueller et al [6] reported that the global numbers for $P$. malariae and $P$. ovale are unknown and the burdens of $P$. ovale and $P$. malariae are even more underrepresented in surveys where microscopy is employed since there are morphological resemblances between $P$. ovale and $P$. vivax.

Non-falciparum malaria may be present in Zambia given that $P$. malariae cases are routinely reported and the anecdotal prevalence is estimated to be approximately $2 \%$. Furthermore, Blossom et al. [7] described a case of a 23-year-old woman who, after a trip to Zambia, presented with a prolonged illness characterized by fevers of up to $38.1^{\circ} \mathrm{C}$ and fatigue. Malaria smear and antibody tests were negative but polymerase chain reaction (PCR) showed the presence of $P$. vivax. Low prevalence of $P$. vivax on the African continent is attributed to high prevalence of the Duffy negative factor, a gene inherent among the majority of indigenous Africans that is otherwise responsible for refractoriness to $P$. vivax [8-10].

Infections with more than one Plasmodium spp. are possible and are called mixed infection. Mixed malaria species infections are often not recognized or are underestimated by microscopists [11,12]. In Asia, surveys usually report that greater than $2 \%$ of infections are mixed, whereas therapeutic studies in $P$. vivax or P. falciparum malaria have demonstrated a high prevalence of up to $30 \%$ of mixed infections. Other malaria species have also been reported during convalescence, suggesting covert coinfections [13]. In Manhica District of Mozambique, sharing borders with Zambia's Eastern Province, P. malariae and $P$. ovale occurred almost exclusively in mixed infections [14]. This situation may be similar to the Zambian scenario as the geography and climatic factors are similar. Although this has not been documented, Zambian technologists and scientists often see and report a few cases of mixed infection routinely.

The Zambia National Malaria Control Programme (NMCP) has been at the forefront of scaling up malaria interventions over the past decade, including the early adoption of artemisinin-based combination therapy and use of rapid diagnostics tests (RDTs) for clinical management of malaria. The 2011-2015 National Malaria Strategic Plan calls for the creation of malaria free zones during its current five-year span. Therefore, understanding the extent to which current tools are able to achieve these goals is important. It follows that further understanding of the distribution of local malaria parasite species is important for developing appropriate preventive as well as diagnostic and treatment option. Unfortunately, many malariaendemic countries, especially in sub-Saharan Africa, are just beginning to understand the relative levels of prevalent malaria parasite species as malaria control programmes begin to expand in the pursuit of malaria elimination. With the anecdotal evidence that $P$. falciparum is the predominant cause of malaria (98\%), Zambia has relied on the exclusive use of histidine-rich protein 2 (HRP2) or P. falciparum-based antigen detection RDTs. [15].

This study sought to determine the proportion of mixed Plasmodium spp. causing malaria in Eastern and Luapula provinces of Zambia using stored samples from the Malaria Indicator Survey of 2012, conducted to monitor the progress of malaria control implementation efforts by the National Malaria Control Program (NMCP). This information is necessary to guide and inform malaria management and control strategies as well as provide country-specific evidence for strategic malaria diagnostic service choices and treatment options.

\section{Methods}

\section{Study design}

Data and samples from MIS conducted between April and May 2012 for two provinces in Zambia were utilized. MIS's have been conducted biannually since 2006 and are used by the NMCP to evaluate progress in scaling up malaria control interventions and to monitor levels of malaria parasite prevalence among children aged five years and younger [16]. The 2012 MIS was the fourth in the series, the first being in 2006, the second in 2008 and the third in 2010. Details of MIS findings are reported elsewhere [17-19].

Study samples from children under six years of age were used from two provinces, Eastern and Luapula. These two provinces were noted to be hyper-endemic for malaria, with the highest levels of malaria parasite prevalence among sampled children during the 2012 MIS study [16]. For this study, all records with complete information on relevant socio-demographic characteristics of household members as well as verifiable records of RDT results, blood smears and dried blood spots (DBS) for all children below six years of age were included in the study. The RDTs used in this survey were HRP2 antigen-based SD Bioline (Standard Diagnostics, INC) RDTs.

\section{Polymerase chain reaction (PCR) protocol}

Identification of Plasmodium spp. was performed using nested PCR, performed at the National Malaria Control 
Centre molecular laboratory with further validation on random samples at Tulane University. DNA was extracted using the chelex method [20] and the nested PCR assay was adapted from Nsobya et al [21].

The protocol was designed such that in the first reaction a conserved region for the four species was amplified (P. falciparum, P. vivax, $P$. ovale and P. malariae). In the second reaction, different primers were run for the four species separately. The band sizes of the products were as follows: P. falciparum $205 \mathrm{bp}$, P. malariae $140 \mathrm{bp}$, P. ovale $800 \mathrm{bp}$ and $P$. vivax $120 \mathrm{bp}$.

The PCR mixture for the first reaction contained $12.5 \mu \mathrm{L}$ of a $2 \mathrm{X}$ master mix containing Taq buffer $(10 \mathrm{mM}$ Tris- $\mathrm{HCl}, \mathrm{pH}$ 8.3, $50 \mathrm{mM} \mathrm{KCL}, 1.5 \mathrm{mM} \mathrm{MgCl} 2), 200 \mu \mathrm{M}$ of each dNTP 2.5 units of Taq DNA polymerase, PCR primers $(10 \mu \mathrm{M}$ of rPLUf and rPLUr) and $5 \mu \mathrm{L}$ of DNA template and water in a final volume of $25 \mu \mathrm{L}$. In the second reaction, the same master mix was used but with different primer concentration $(10 \mu \mathrm{M}$ of each of the species-specific primers in separate tubes), $2 \mu \mathrm{L}$ DNA template from the first reaction in a $25 \mu \mathrm{L}$ reaction volume. The PCR was run in a gene Amp PCR system 9700 (Applied Biosystems thermocycler, Foster City CA, USA) under the following conditions: the first reaction had denaturation at $94^{\circ} \mathrm{C}$ for one minute followed by 35 cycles at $94^{\circ} \mathrm{C}$ for one minute, $58^{\circ} \mathrm{C}$ for two minutes and $72^{\circ} \mathrm{C}$ for five minutes; the second reaction (with four tubes for each primer) had denaturation at $94^{\circ} \mathrm{C}$ for one minute, followed by 30 cycles at $94^{\circ} \mathrm{C}$ for one minute, $58^{\circ} \mathrm{C}$ for two minutes and $72^{\circ} \mathrm{C}$ for five minutes. The final cycle was followed by an extension time of five minutes at $72^{\circ} \mathrm{C}$. A $2 \%$ agarose gel stained with ethidium bromide was run for visualisation of the PCR product [20].

\section{Data and statistical analysis}

Demographic and laboratory data of the participants' records was analysed with Stata version 11 (College Station, Texas, USA) with the cluster effect accounted for in the analyses. The Mantel-Haenszel chi square test $\left(x^{2}\right)$, and in some cases Fisher's exact tests for proportions, were used to test for independence. Multivariate logistic regression was used to examine the association between background factors with malaria between comparable categories. The distribution of age as a continuous variable conformed to normality as assessed by probability plots.

\section{Ethical approval}

The 2012 MIS survey protocol received clearance by the University of Zambia Biomedical Research Ethics Committee as a continuation of the 2006, 2008 and 2010 surveys. The reference for the Malaria Indicator Survey it was 002-03-12. In addition, participation in the MIS questionnaires and malaria testing were based on informed consent from their respective parents or guardians as all children in the survey were below six years of age. Respondents were counselled and informed that the testing was purely for research purposes and was to be handled anonymously. However, respondents were informed of their test results and available treatment was provided based on national malaria treatment standard care guidelines. For this study of mixed infections, additional approval was sought from the committee; reference number was 001-10-12.

\section{Results}

\section{Socio-demographic characteristics}

The de facto eligible population comprised only children younger than six years who had complete records and were tested for malaria infection. Thus, only 873 children were included in the final analysis. Of these children, $47 \%$ were male and $53 \%$ were female. There were 504 (57.7\%) children from Eastern Province and 369 (42.3\%) from Luapula Province. Larger proportions (95.7\%) of these children were classified according to the national census as living in rural clusters, with only $4.4 \%$ living in an urban cluster (Table 1).

\section{Prevalence}

Overall, the prevalence of malaria infection using PCR was 54.3\% (95\% CI 51-57.6\%) (Figure 1), with Luapula Province accounting for a higher burden than Eastern Province $(55.6 \%$ vs. $52.6 \%$, respectively). From the total positive samples, the predominant species was $P$. falciparum (88.3\%) with $11.6 \%$ due to non-falciparum species and $10.3 \%$ were mixed infections (Table 2). The observed mixed infections were in five categories or combinations. Most children were found to have a combination of $P$.falciparum and P. malariae (6.5\%); P. falciparum, P. malariae and $P$. ovale (6\%); and P. falciparum and $P$. ovale (2.1\%). The combinations of $P$. falciparum, $P$. malariae and $P$. vivax (0.2\%), and $P$. falciparum and $P$. vivax $(0.2 \%)$ were rare (Table 2).

\section{Factors associated with mixed plasmodium infections}

Assessment of factors associated with mixed Plasmodium infections (Table 3) showed an association between mixed infections with province and age of the child. Children older than two years of age were almost three times more likely to have mixed malaria infections than children younger than two years of age (aOR 2.8, 95\% CI, 1.31-5.69). This association was stronger in Luapula province where likelihood of children having mixed infection was four-fold higher (aOR 4.2, 95\% CI 2.00-8.73) than in children residing in Eastern Province.

\section{Discussion}

The study showed a significantly high prevalence of malaria in two provinces of Zambia, demonstrating for the 
Table 1 Socio-demographic factors for the study participants in the Luapula and Northern Provinces in Zambia

\begin{tabular}{|c|c|c|}
\hline \multicolumn{3}{|c|}{ Total number of children test $=873$} \\
\hline Characteristics & $\mathbf{N}$ & $\%(\mathrm{Cl})$ \\
\hline \multicolumn{3}{|l|}{ Gender } \\
\hline Male & 410 & $47.0(43.6-50.2)$ \\
\hline Female & 463 & $53.0(49.7-56.4)$ \\
\hline \multicolumn{3}{|l|}{ Age of children } \\
\hline$<1$ year & 132 & $15.1(12.7-17.5)$ \\
\hline $1 \mathrm{yr}$ & 144 & 16.5(14.0-19.0) \\
\hline $2 y r s$ & 151 & 17.3(14.8-19.8) \\
\hline 3 yrs & 178 & 20.4(17.7-23.1) \\
\hline 4 yrs & 156 & $17.9(15.3-20.4)$ \\
\hline $5 \mathrm{yrs}$ & 112 & 12.8(10.6-15.1) \\
\hline \multicolumn{3}{|l|}{ Province } \\
\hline Eastern & 504 & $57.7(54.4-61.0)$ \\
\hline Luapula & 369 & $42.3(39.0-45.5)$ \\
\hline \multicolumn{3}{|l|}{ Residence } \\
\hline Urban & 38 & $4.4(3.0-5.7)$ \\
\hline Rural & 835 & 95.7 (94.3-97.0) \\
\hline Age of Mother & 47 & $6.0(4.3-7.7)$ \\
\hline $15-19$ & 207 & $26.4(23.3-29.5)$ \\
\hline $20-24$ & 203 & $25.9(23.0-28.9)$ \\
\hline $25-29$ & 254 & $32.4(29.1-35.6)$ \\
\hline 30-39 & 74 & $9.4(7.4-11.5)$ \\
\hline \multicolumn{3}{|l|}{ School level of mother } \\
\hline Low-Below grade 5 & 420 & $53.5(50.0-57.0)$ \\
\hline High- Grade 5 and above & 365 & $46.5(43.0-50.0)$ \\
\hline
\end{tabular}

first time to our knowledge the prevalence of the four major Plasmodium species in the studied population. Although the malaria prevalence is predominantly due to $P$. falciparum, the prevalence of non-falciparum mixed infections was significantly higher than previously reported.

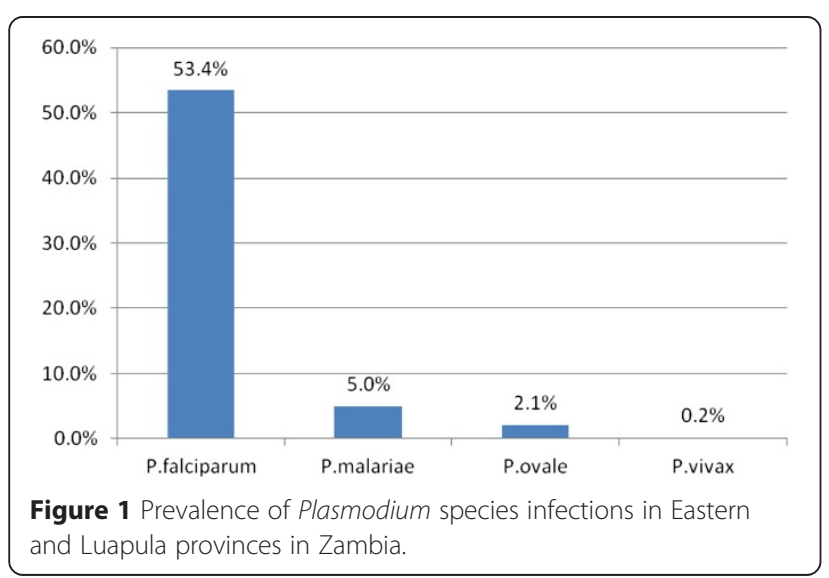

Table 2 Differential overall prevalence of mixed and mono infections in Eastern and Luapula Provinces combined

\begin{tabular}{lll}
\hline Infection pattern & No. & Percentage \\
\hline P.f & 419 & 88 \\
P.m & 4 & 1 \\
P.o & 2 & 0.4 \\
Pf + Pm + Po & 6 & 1.3 \\
Pf + Pm + Pv & 1 & 0.2 \\
Pf + Pm & 31 & 6.5 \\
Pf + Po & 10 & 2.1 \\
Pf + Pv & 1 & 0.2 \\
Total & 474 & 100 \\
Total combinations & 55 & $10.3^{*}$ \\
\hline
\end{tabular}

Note: All positives is the $n=474,{ }^{*} 10.3 \%$, is the percentage for all mixed infection combination.

Key: Pf-Plasmodium falciparum; Pm- Plasmodium malariae; Po- Plasmodium ovale; Pv- Plasmodium vivax.

This high prevalence partly explains why malaria still remains one of the leading causes of morbidity and mortality in Zambia despite the current control and management measures. The high prevalence of malaria due to all four species and in both mono and mixed infections patterns clearly reveals not just the presence of non-falciparum species but also the dynamic and evolving nature of the malaria epidemic. The $11.6 \%$ is actually close to the anecdotal level of non-falciparum-only infections known, but

Table 3 Results logistic regression model for malaria mixed infection for the study participants from Luapula and Northern Provinces in Zambia

\begin{tabular}{llll}
\hline $\begin{array}{l}\text { Malaria mixed parasites infection } \\
\text { Characteristic }\end{array}$ & $\begin{array}{l}\mathbf{n}=\mathbf{8 7 3} \\
\text { Prevalence }\end{array}$ & aOR & $\mathbf{9 5 \%} \mathbf{C l}$ \\
\hline $\begin{array}{l}\text { Age in years } \\
0>2\end{array}$ & $2.8 \%$ & 1 & \\
$2-5$ & $8.3 \%$ & 2.80 & $1.31-5.69$ \\
Gender & & & \\
Male & $46.4 \%$ & 1 & \\
Female & $53.6 \%$ & 0.9 & $0.50-1.88$ \\
Age of the mother in years & & & \\
15-24 & $3.5 \%$ & 1 & \\
25-39 & $5.3 \%$ & 1.4 & $0.61-3.13$ \\
40-49 & $8.1 \%$ & 2.6 & $0.83-7.84$ \\
Province & & & \\
Eastern & $3.0 \%$ & 1 & \\
Luapula & $9.2 \%$ & 4.2 & $2.00-8.73$ \\
Ownership of Mosquito net & & & \\
No & $58.0 \%$ & 1 & \\
Yes & $53.4 \%$ & 0.5 & $0.14-1.79$ \\
\hline
\end{tabular}


mixed falciparum infections account for a more significant contribution of the total infection prevalence than previously considered. These findings suggest that there is need for continued monitoring of non-falciparum infection prevalence in this population so as to decide when species-specific RDTs should be introduced for diagnostic purposes. The species-specific diagnosis will be important when the prevalence of malaria in this population reduces and as the country moves towards malaria elimination. In addition, Coartem, the first-line treatment drug in Zambia, does not clear Plasmodium hypnozoites and radical treatment with primaquine, effective against hypnozoites may play an important role in the control and eventually elimination of vivax and ovale malaria, and malaria consequently [22].

Differential species burden patterns observed were not surprising as similar observations have been reported elsewhere [14,23]. However, the fact that non-falciparum malaria cases were present in the study population necessitates the need to have diagnostic tools or approaches at the national level that should facilitate the detection of such non-falciparum malaria cases. While it should not necessarily be the primary diagnostic tool, diagnostic approaches that have the capacity to further investigate and detect non-falciparum malaria may become important in future. For health facilities where microscopy services are present, there is a need to conduct refresher courses for laboratory staff and microscopists with an emphasis on Plasmodium species-wide microscopy so as to strengthen non-falciparum malaria diagnosis as has been reported and advocated elsewhere $[7,10,24]$.

Among the factors associated with mixed malaria infections, age was found to be a predictor. This is in agreement with a similar study by Guerra-Neira et al [25] who reported an inverse correlation between of age and multiplicity of Plasmodium infection, with children under five years of age having higher frequency of mixed Plasmodium malaria compared to individuals older than five years of age. Children younger than two years in our study were found to be less likely to have mixed malaria infections compared to those aged from two to five years. This could be partly explained by the maternally derived antibodies believed to offer protection from infections. Riley et al reported that neonates and infants are relatively protected from clinical malaria, although the mechanism of the protection is not clearly understood [26]. Indeed, the dynamics of immunity in neonates, infants and toddlers is quite remarkable resulting in variable susceptibility to infection $[27,28]$. Noteworthy here is that, unlike the study by Guerra-Neira et al [25], our study population was limited to children younger than five years of age. In addition, these findings could be attributed to the use of insecticide-treated nets (ITNs) in that younger children tend to sleep under
ITNs as breast-feeding infants but, at the age of two-tothree years, they are weaned off from breasting feeding and may not sleep under an ITN.

\section{Conclusion}

This study reveals the presence of mixed infections in two provinces of Zambia with an overall prevalence of $10.3 \%$. The major factor associated with mixed infection was the age of the child. This might call for repackaging of control and management measures at national level. The presence of non-falciparum infections might vary with transmission patterns, demographic trends and geographical contrasts. There is the need for these to be monitored through a functional surveillance system so as to understand the epidemiological profile. Given that most of the non-falciparum malaria cases occur as mixed infections, the use of HRP2-based RDTs can still continue in health facilities but need to be strengthened with improved and enhanced microscopy to facilitate detection of the non-falciparum malaria cases. Finally, there is a need for further studies to have a complete, detailed profile and epidemiology of non-falciparum species of malaria in the country, especially in the era of malaria elimination activities in the country and region.

\section{Abbreviations}

HRP2: Histidine rich protein 2; DBS: Dried Blood Spot; DNA: Deoxyribonucleic Acid; ITNs: Insecticide-treated nets; NMCC: National Malaria Control Centre; NMCP: National Malaria Control Programme; MIS: Malaria Indicator Survey; PCR: Polymerase chain reaction; RDTs: Rapid diagnostic tests; WHO: World Health Organisation.

\section{Competing interests}

The author declares that they have no competing interests.

\section{Authors' contributions}

LS Principle investigator-designed the study, carried out the molecular analysis, drafted the manuscript; JC contributed to the study design, data analysis, writing and correction of the manuscript; gave approval for submission; MH played a role in the running of the PCR samples, he was involved in correction of the manuscript; JM played a major role in acquisition of the data, data cleaning, analysis and manuscript writing; NK facilitated independent validation of mixed infections, supported the molecular laboratory analysis of study and corrected the manuscript; WM supported the molecular laboratory analysis, and was involved in the writing and correction of the manuscript; CM was also involved in designing the study and data analysis, corrected the manuscript and gave approval for submission of the manuscript. All authors read and approved the final manuscript.

\section{Authors' information}

LS-Lungowe Sitali is a Medical Parasitologist and an Epidemiologist with an MSC and a BSc in Biological Sciences, and is a lecturer at the University of Zambia; JC-James Chipeta is a Senior Lecturer and Honorary Consultant Paediatrician, and is Assistant Dean Research for the School of Medicine; MH-Moonga B. Hawela is a Medical Parasitologist with an MSC and a BSC in Biomedical Sciences, and he is the chief Parasitologist at the Zambia National Malaria Control Centre; JM-John M. Miller is a PhD holder, an Epidemiologist and a malaria surveillance specialist for the Malaria Control and Elimination Partnership in Africa (MACEPA) in Zambia; NK- Nirbhay Kumar is Professor and the Department of Tropical Medicine and Director of Vector-Borne Infectious Diseases Research Centre, Tulane University School of Public Health and Tropical Medicine; WM is a Paediatrician with subspecialty training in infectious diseases based at John Hopkins Malaria Research Institute; CM- Charles Michelo is an Associate 
Professor of Epidemiology and Head of Department of the University of Zambia School of Medicine, Department of Public Health.

\section{Acknowledgments}

This work was supported by the following organisations: Norwegian Agency for Development Cooperation (NORAD)'s Programme for Master Studies, project number 2010/12841, which is a tripartite cooperation between the University of Bergen, Norway, College of Medicine in Malawi and the Department of Public Health, University of Zambia. We also acknowledge the major role played by the PATH- Malaria Control and Elimination Partnership in Africa (MACEPA); Division of Microbiology and Infectious Diseases, National Institute of Allergy and Infectious Diseases, National Institute of Health as part of the International Centres of Excellence in Malaria Research (ICEMR); and Fogarty international, National Institute of Health Training grant, the Malaria Training Capacity Building Southern Africa (MTCBSA grant number 2D43TW001587-06A2) programme. We are grateful to the Ministry of Health and the National Malaria Control Centre that allowed us to use the MIS data. Finally we wish to acknowledge Mulenga Hope Mwenda and Kasanda Chiwela who helped in running the PCR samples.

\section{Author details}

${ }^{1}$ Department of Biomedical Science, University of Zambia, School of Medicine, Lusaka, Zambia. ${ }^{2}$ Department of Paediatrics and Child Health, School of Medicine, Malaria Research Unit (SMUTH-MRU), Lusaka, Zambia. ${ }^{3}$ Department of Paediatrics and Child Health, University of Zambia, School of Medicine, Lusaka, Zambia. ${ }^{4}$ PATH-Malaria Control and Elimination Partnership in Africa (MACEPA), National Malaria Control Centre, Lusaka, Zambia. ${ }^{5}$ Ministry of Health, National Malaria Control Centre, Lusaka, Zambia. ${ }^{6}$ Department of Tropical Medicine, Tulane University School of Public Health and Tropical Medicine, New Orleans, USA. ${ }^{7}$ John Hopkins Malaria Research Institute, Bloomberg School of Public Health, Johns Hopkins University, Baltimore, MD, USA. ${ }^{8}$ Department of Public Health, University of Zambia, School of Medicine, Lusaka, Zambia.

Received: 8 December 2014 Accepted: 22 April 2015

Published online: 02 May 2015

\section{References}

1. WHO news release, 2011 http://wnw.who.int/malaria/world_malaria_report_2011/ en/ (Accessed on 10 October 2013).

2. World Health Organisation report, World Malaria Report 2012 http;// www.who.int/malaria/publication/world_malaria_report_2012/report/en (Accessed on 12 October 2013).

3. Carter R, Mendis KN. Evolutionary and historical aspects of the burden of malaria. Clin Microbiol Rev. 2002;15:564-94.

4. Gething PW, Elyazar IR, Moyes CL, Smith DL, Battle KE, Guerra CA, et al. A long neglected world malaria map: plasmodium vivax endemicity in 2010. PLoS Negl Trop Dis. 2012;6(9):e1814.

5. Hay SI, Okiro EA, Gething PW, Patil AP, Tatem AJ, Guerra CA, et al. Estimating the global clinical burden of Plasmodium falciparum malaria in 2007. PLoS Med. 2010;7(6):e1000290. Epub 2010/06/22.

6. Mueller I, Zimmerman PA, Reeder JC. Plasmodium malariae and Plasmodium ovale — the "bashful" malaria parasites. Trends Parasitol. 2007;6:278-83.

7. Blossom BD, Kings $\mathrm{CH}$, Armitage KB. Occult plasmodium vivax infection diagnosed by a polymerase chain reaction-based detection system: a case report. The Am Soc Trop Med Hyg. 2005;73(1):188-90.

8. Carvalho TL, Ribolla PE, Curi RA, Moto LS. Characterization and transcriptional analysis of the promoter region of the Duffy blood group, chemokine receptor(DARC) gene in cattle. Vet Immunol Immunopathol. 2009;132:153-9.

9. Mendes C, Dias F, Figueiredo J, Mora VG, Cano J, de Sousa B, et al. Duffy negative antigen is no longer a barrier to plasmodium vivax - molecular evidences from the African west coast (Angola and Equatorial Guinea). PLoS Negl Trop Dis. 2011;5(6):1192.

10. Larina SN, Sakharova TV and Chebyshev NV. Genetic resistance to malaria. Med Parazitol (Mosk) 2009, 10-4. Ncbi.nim.gov/pubmed/19566055.

11. Mbakilwa H, Manga C, Kibona S, Mtei F, Meta J, Shoo A, et al. Quality of malaria microscopy in 12 district hospital laboratories in Tanzania. Pathog Glob Health. 2012;106(6):330-4. Epub 2012/11/28.
12. Frean J, Perovic O, Fensham V, McCarthy K, von Gottberg A, de Gouveia L, et al. External quality assessment of national public health laboratories in Africa, 2002-2009. Bull World Health Organ. 2012;90(3):191-9A. Epub 2012/03/31.

13. Mayxay M, Pukrittayakame S, Newton PN, White NJ. Mixed-species malaria infections in humans. Trends Parasitol. 2004;20(5):233-40.

14. Marques P, Saúte F, Pinto W, Cardoso S, Pinto J, Alonso PL, et al. Plasmodium species mixed infections in two areas of Manhiça District. Mozambique Int J Biol Sci. 2005;1(3):96-102.

15. Ministry of Health. Guidelines for the Diagnosis and Treatment of Malaria in Zambia, 2010 Edition, Lusaka, Zambia Ministry of Health; 2010 (http:// www.nmcc.org.zm/files/GuidelinesonDiagnosisandTreatmentofMalariain Zambia_4thEd_2-24-14.pdf).

16. Ministry of Health. Zambia National Malaria Indicator Survey 2012, Lusaka. (http:// www.nmcc.org.zm/files/FullReportZambiaMIS2012_July2013_withsigs2.pdf).

17. Ministry of Health. Zambia National Malaria Indicator Survey 2010, Lusaka. (http://www.nmcc.org.zm/files/FullReportZambiaMIS2010_001.pdf).

18. Ministry of Health. Zambia National Malaria Indicator Survey 2008, Lusaka. (http://www.nmcc.org.zm/files/ZambiaMIS2008Final.pdf).

19. Ministry of Health. Zambia National Malaria Indicator Survey 2006 draft report (http://www.nmcc.org.zm/files/2006_Zambia_Malaria_Indicator_Survey.pdf).

20. Kain KC, Lanar DE. Determination of genetic variation within $P$. falciparum by using enzymatically amplified DNA from filter paper disks impregnated with whole blood. J Clin Microbiol. 1991;29:1171-4.

21. Nsobya SL, Parikh S, Kironde F, Lubega G, Kamya MR, Rosenthal PJ, et al. Molecular evaluation of the natural history of asymptomatic parasitemia in Ugandan children. J Infect Dis. 2004;189:2220-6.

22. White $\mathrm{JN}$. The role of anti-malarial drugs in eliminating malaria. Malar J. 2008;7(1):S8. doi: 10.1186/1475-2875-7-S1-S8.

23. Zakeri S, Kakar Q, Ghasemi F, Raeisi A, Butt W, Safi N, et al. Detection of mixed plasmodium falciparum \& P. Vivax infections by nested-PCR in Pakistan, Iran \& Afghanistan, Indian. J Med Res. 2010;132:31-5.

24. Ohrt C, Obare P, NanaKorn A, Adhiambo C, Awuondo K, O'Meara PW, et al. Establishing a malaria diagnosis centre of excellence in Kisumu. Kenya Malar J. 2007;6:79.

25. Guerra-Neira A, Rubio JM, Royo JR, Ortega JC, Aunon AS, Diaz PB, et al. Plasmodium diversity in non-malaria individuals from the Bioko Island in Equatorial Guinea (West Central-Africa). Int J Health Geogr. 2006;5:27. Epub 2006/06/21.

26. Riley EM, Wagner GE, Akanmori BD, Koram KA. Do maternally acquired antibodies protect infants from malaria infection? Parasite Immunol. 2001;23:51-9.

27. Doolan DL, Doban C, Baird JK. Acquired immunity to malaria clinical. Microbiol Rev. 2009;22(1):13-36.

28. Moormann AM. How might infant and paediatric immune responses influence malaria vaccine efficacy? Parasite Immunol. 2009;31:547-59.

\section{Submit your next manuscript to BioMed Central and take full advantage of:}

- Convenient online submission

- Thorough peer review

- No space constraints or color figure charges

- Immediate publication on acceptance

- Inclusion in PubMed, CAS, Scopus and Google Scholar

- Research which is freely available for redistribution

Submit your manuscript at www.biomedcentral.com/submit

C) BioMed Central 